\title{
Effect of physical training on mitochondrial respiration and reactive oxygen species release in skeletal muscle in patients with obesity and type 2 diabetes
}

\author{
M. Hey-Mogensen • K. Højlund • B. F. Vind • L. Wang • \\ F. Dela • H. Beck-Nielsen • M. Fernström • K. Sahlin
}

Received: 23 March 2010/Accepted: 13 April 2010 /Published online: 6 June 2010

(C) Springer-Verlag 2010

\begin{abstract}
Aim/hypothesis Studies have suggested a link between insulin resistance and mitochondrial dysfunction in skeletal muscles. Our primary aim was to investigate the effect of aerobic training on mitochondrial respiration and mitochondrial reactive oxygen species (ROS) release in skeletal muscle of obese participants with and without type 2 diabetes.

Methods Type 2 diabetic men $(n=13)$ and control $(n=14)$ participants matched for age, BMI and physical activity completed 10 weeks of aerobic training. Pre- and posttraining muscle biopsies were obtained before a euglycaemichyperinsulinaemic clamp and used for measurement of respiratory function and ROS release in isolated mitochondria. Results Training significantly increased insulin sensitivity, maximal oxygen consumption and muscle mitochondrial respiration with no difference between groups. When expressed in relation to a marker of mitochondrial density (intrinsic mitochondrial respiration), training resulted in
\end{abstract}

M. Hey-Mogensen $(\bowtie) \cdot$ F. Dela

Center for Healthy Aging, Department of Biomedical Sciences,

University of Copenhagen,

Blegdamsvej 3B,

2200 Copenhagen, Denmark

e-mail: mmogensen@sund.ku.dk

K. Højlund · B. F. Vind · H. Beck-Nielsen

Diabetes Research Centre, Department of Endocrinology,

Odense University Hospital,

Odense, Denmark

M. Hey-Mogensen $\cdot$ K. Sahlin

Institute of Sports Science and Clinical Biomechanics,

University of Southern Denmark,

Odense, Denmark

L. Wang $\cdot$ M. Fernström $\cdot$ K. Sahlin

Stockholm University College of P.E. and Sports, GIH,

Stockholm, Sweden increased mitochondrial ADP-stimulated respiration (with NADH-generating substrates) and decreased respiration without ADP. Intrinsic mitochondrial respiration was not different between groups despite lower insulin sensitivity in type 2 diabetic participants. Mitochondrial ROS release tended to be higher in participants with type 2 diabetes.

Conclusions/interpretation Aerobic training improves muscle respiration and intrinsic mitochondrial respiration in untrained obese participants with and without type 2 diabetes. These adaptations demonstrate an increased metabolic fitness, but do not seem to be directly related to training-induced changes in insulin sensitivity.

Keywords Exercise - Gene expression - Insulin sensitivity · Mitochondrial respiration - Reactive oxygen species .

Type 2 diabetes $\cdot$ UCP3

$\begin{array}{ll}\text { Abbreviations } \\ \mathrm{CS} & \text { Citrate synthase } \\ \mathrm{CS}_{\text {mito }} & \text { CS activity in the mitochondrial rich solutions } \\ \mathrm{CS}_{\text {muscle }} & \text { CS activity per g muscle wet weight } \\ \mathrm{ETC} & \text { Electron chain flux } \\ \mathrm{MHC} & \text { Myosin heavy chain } \\ \mathrm{RCI} & \text { Respiratory control index } \\ R_{\mathrm{d}} & \text { Rate of glucose disposal } \\ \mathrm{ROS} & \text { Reactive oxygen species } \\ \mathrm{ROS}_{\text {anti }} & \text { Mitochondrial ROS release in the presence of } \\ & \text { antimycin A } \\ \mathrm{ROS}_{\text {rot }} & \begin{array}{l}\text { Mitochondrial ROS release in the presence of } \\ \text { rotenone }\end{array} \\ \mathrm{State} & \text { ADP-stimulated respiration } \\ \mathrm{State} 4 & \text { Respiration without ADP } \\ \mathrm{U} & \text { Unit } \\ \mathrm{UCP} & \text { Uncoupling protein isotype } 3 \\ \dot{V} \mathrm{O}_{2} \text { peak } & \text { Maximal oxygen consumption }\end{array}$




\section{Introduction}

Type 2 diabetes and obesity are characterised by skeletal muscle insulin resistance. Insulin resistance precedes the development of type 2 diabetes and is seen in non-diabetic first-degree relatives of patients with type 2 diabetes [1]. Despite intense research, the mechanisms underlying insulin resistance are not completely understood.

A decade ago Kelley and Mandarino suggested that insulin resistance in skeletal muscles is caused by mitochondrial dysfunction [2]. Since then several studies have investigated this hypothesis, but so far results have been ambiguous. When mitochondrial activity was determined per muscle weight (i.e. muscle oxidative capacity), studies have either shown no differences [3-5] or a lower [6-12] mitochondrial activity in insulin-resistant participants. Studies that have related mitochondrial activity to markers of mitochondrial volume (intrinsic mitochondrial activity) have either shown a lower $[6,12,13]$ or no difference $[9$ $11,14,15]$ in mitochondrial activity in insulin-resistant participants. Thus, reduced insulin sensitivity in insulinresistant participants is not consistently related to low mitochondrial activity.

Recently, it was suggested that beside the potential link between mitochondrial activity and insulin sensitivity, the mitochondrial production of reactive oxygen species (ROS) may also play an essential role in determining skeletal muscle insulin sensitivity. In cultured cells there is experimental evidence that ROS have an inhibitory effect on insulin signalling $[16,17]$. Human studies have shown that obesity and high-fat diet increase mitochondrial hydrogen peroxide release [18]. Furthermore, a recent study showed that mitochondrial ROS release was higher in muscle of patients with type 2 diabetes than in obese controls, but similar to that observed in lean control participants [19]. Interestingly, studies have also shown that a transient physiological increase in ROS may be essential for a training-induced increase in insulin sensitivity in type 2 diabetes patients [20] and for protection against high-fat diet-induced insulin resistance in rodents [21]. Both these studies $[20,21]$ suggest that ROS have a beneficial effect when increased ROS is transient, but are harmful when the increased concentration is sustained, as seen in response to chronic hyperglycaemia and dyslipidaemia.

As the major site for cellular ROS production, the mitochondrion [22] is especially prone to oxidative damage. The higher mitochondrial ROS release in type 2 diabetes patients [19] may explain the lower intrinsic mitochondrial activity seen in some studies $[6,12,13]$. It is, however, possible that mitochondrial ROS release is influenced by the level of physical fitness [23], which was not assessed in the previous studies [19]. Thus, it remains to be established whether mitochondrial ROS production differs between type 2 diabetes and control participants who are matched for physical fitness.

It is well known that physical training increases skeletal muscle insulin sensitivity in type 2 diabetes patients [24]. The mechanism might be related to improved muscle oxidative capacity [25]. A recent study showed that physical training improves in vivo mitochondrial function concomitantly with increased insulin sensitivity in type 2 diabetes patients and control participants [26]. As suggested by the authors, the improved in vivo mitochondrial function may be due to increased mitochondrial content and/or increased functionality per mitochondrion (intrinsic) [26]. These results are in agreement with another study showing that changes in plasma glucose concentration and $\mathrm{HbA}_{1 \mathrm{c}}$, induced by increased physical activity and weight-loss, were significantly associated with increased mitochondrial content [27]. Interestingly, it has been suggested that insulin-resistant participants may have a lower response to physical training [28]. Recently it was shown that the response to acute exercise in terms of expression and protein content of genes coding for mitochondrial biogenesis was lower in insulin-resistant non-diabetic participants than in lean control participants [29]. However, it remains to be investigated whether the response to physical training in genes coding for mitochondrial biogenesis is lower in insulin-resistant participants. It also remains to be assessed whether physical training alters intrinsic mitochondrial function in type 2 diabetes patients and control participants.

The purpose of the present study was to investigate the effect of training on mitochondrial ROS release, mitochondrial respiration (both intrinsic and per tissue weight) and markers of mitochondrial biogenesis in skeletal muscle of obese type 2 diabetes patients and obese non-diabetic control participants. We also sought to examine the relation of training to concomitant changes in insulin sensitivity. We hypothesised that type 2 diabetes patients (vs obese controls) would have higher mitochondrial ROS release, but lower intrinsic mitochondrial respiration, and that these abnormalities would be improved after endurance training.

\section{Methods}

Participants We studied 13 obese type 2 diabetes patients and 14 obese control participants. Control participants had normal glucose tolerance (by 120 min OGTT with $75 \mathrm{~g}$ carbohydrate) and no family history of type 2 diabetes. Type 2 diabetes patients and control participants were matched for age (45-65 years), sex (all men) and obesity $\left(\mathrm{BMI}>27 \mathrm{~kg} / \mathrm{m}^{2}\right.$ ). Type 2 diabetes patients were treated with diet alone $(n=1)$ or diet in combination with sulfonylurea $(n=5)$ or metformin $(n=11)$. The patients were all negative for GAD65 antibody and had no signs of 
diabetic retinopathy, neuropathy, nephropathy or macrovascular complications. All participants had normal results on blood tests screening for hepatic and renal function. All participants gave written informed consent and the study was approved by the local Ethics Committee of Funen and Vejle County, Denmark. It was performed in accordance with the Declaration of Helsinki.

All participants completed the pre-training tests (see below), except one control participant who was unable to complete the euglycaemic-hyperinsulinaemic clamp test. Three participants did not complete the post-training test due to complete interruption of the training programme (one person per group) or due to unwillingness to repeat the clamp test (one control). Despite dropouts, all participants who completed the pre-training tests were included in the data analysis to increase statistical power for the comparison of mitochondrial ROS release between type 2 diabetes and control participants.

Training programme Participants underwent 10 weeks of aerobic training as described previously [30]. Briefly, training consisted of 10 weeks of cycling on stationary bikes. Training was partly supervised (two times per week) and partly done at home (two to three times per week). Training sessions ranged from 20 to $35 \mathrm{~min}$ per session and were performed four to five times per week at an average exercise intensity of $\sim 65 \%$ of maximal oxygen consumption $\left(\dot{V} \mathrm{O}_{2 \text { peak }}\right)$. Participants used heart rate monitors (Polar Vantage; Polar Electro, Kempele, Finland) during all sessions. Data recorded by the heart rate monitors were used to verify training intensity and training volume. Participants were instructed not to make any dietary changes throughout training programme.

Test protocols Before and after the 10-week training, participants underwent an exercise test to determine $\dot{V} \mathrm{O}_{2 \text { peak }}$ and a euglycaemic-hyperinsulinaemic clamp, combined with muscle biopsies as described previously [30]. Briefly, the euglycaemic-hyperinsulinaemic clamp was performed before and after the 10 week training programme, $\sim 48 \mathrm{~h}$ after the last exercise bout. In patients with type 2 diabetes, medication was withdrawn 1 week prior to the clamp studies and then resumed. Participants underwent a $2 \mathrm{~h}$ basal tracer equilibration period followed by $3 \mathrm{~h}$ of insulin infusion at $80 \mathrm{mU} \mathrm{min}{ }^{-1} \mathrm{~m}^{-2}$ after a $12 \mathrm{~h}$ overnight fast. Plasma glucose was allowed to decline to $\sim 5.5 \mathrm{mmol} / 1$ in diabetic participants before glucose infusion was initiated. The insulinstimulated rate of glucose disposal $\left(R_{\mathrm{d}}\right)$ was calculated as the average amount of glucose ( $\left.\mathrm{mg} \mathrm{\operatorname {min } ^ { - 1 }}[\mathrm{kg} \text { fat-free-mass }]^{-1}\right)$ needed to maintain euglycaemia $(\sim 5.5 \mathrm{mmol} / \mathrm{l})$ during the last $20 \mathrm{~min}$ of the clamp.

Muscle biopsies from vastus lateralis muscle were obtained on the day of the clamp test, but before insulin infusion, using a modified Bergström needle with suction under local anaesthesia $(10 \mathrm{ml}$ lidocaine $2 \%)$. The major part of the biopsy was used for isolation of mitochondria as described previously [31]. The remaining part was rapidly frozen in liquid nitrogen and stored at $-80^{\circ} \mathrm{C}$ for later determination of uncoupling protein isotype 3 (UCP3) protein content, enzyme activity, fibre-type distribution and measures of gene expression.

Respiratory activity of isolated mitochondria The intrinsic mitochondrial respiratory function $\left(\mathrm{nmol} \mathrm{O}_{2} \min ^{-1}[\mathrm{U}\right.$ of citrate synthase (CS) activity] ${ }^{-1}$ ) and respiration per kg wet weight $\left(\mathrm{mmol} \mathrm{O}_{2} \mathrm{~min}^{-1}\left[\mathrm{~kg}\right.\right.$ wet weight $\left.{ }^{-1}\right)$ was determined by measuring oxygen consumption (DW1 Oxygraph; Hansatech Instruments, King's Lynn, UK) in isolated mitochondria at $25^{\circ} \mathrm{C}$. The following two protocols were used (Fig. 1) with substrates being added successively:

- Protocol 1: (1) ADP-stimulated respiration (State3) $(0.3 \mathrm{mmol} / \mathrm{l} \mathrm{ADP})$ and respiration without ADP (State4) (non-coupled respiration) with pyruvate $(5 \mathrm{mmol} / \mathrm{l})$ and L-malate ( $2 \mathrm{mmol} / \mathrm{l})$ (State $3_{\text {PYRUVATE}}$, State $\left.4_{\text {PYrUVATE }}\right)$; (2) State3 respiration after further addition of glutamate (4 mmol/l) (State3 $3_{\text {PYRUVATE+GLUTAMATE}}$ ); and (3) maximal respiration through permeabilised mitochondria after addition of cytochrome $c(2 \mu \mathrm{mol} / \mathrm{l})$, alamethicin $(6 \mu \mathrm{g} / \mathrm{ml})$ and $\mathrm{NADH}$ as direct substrate to complex I $(0.4 \mathrm{mmol} / \mathrm{l})$ (electron transport chain flux [ETC $\left.]_{\max }\right)$.

- Protocol 2: (1) State3 and State4 with palmitoyl-Lcarnitine $(10 \mu \mathrm{mol} / \mathrm{l})$ and L-malate $(2 \mathrm{mmol} / \mathrm{l})$

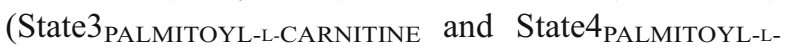
CARNITINE); (2) State3 respiration after further addition of succinate $(6 \mathrm{mmol} / \mathrm{l})$ (State $3_{\text {PALMITOYL-L-CARNITINE+ }}$ SUCCINATE); and (3) State3 respiration with multiple substrates (complex I- and II-related substrates) after addition of pyruvate and glutamate (State $3_{\text {MULTI }}$ ).

Due to lack of material or low respiratory activity, Protocol 2 and State4 respiration in Protocol 1 before training were not performed in eight (control $n=5$, type 2 diabetes $n=3$ ) and seven (control $n=5$, type 2 diabetes $n=2$ ) participants, respectively.

Respiratory control index (RCI) (=State3 divided by State4) and ratio of ADP consumed:oxygen utilised (i.e. ADP added divided by oxygen consumption during State3) were calculated when using pyruvate+malate and palmitoyl-L-carnitine+malate, and used to evaluate the success of the isolation procedure, and the integrity and coupled state of inner mitochondrial membrane.

Mitochondrial ROS release Mitochondrial hydrogen peroxide $\left(\mathrm{H}_{2} \mathrm{O}_{2}\right)$ release (nmol $\mathrm{H}_{2} \mathrm{O}_{2} \min ^{-1}$ [U CS activity] ${ }^{-1}$ ) was measured in isolated mitochondria with Amplex Red 

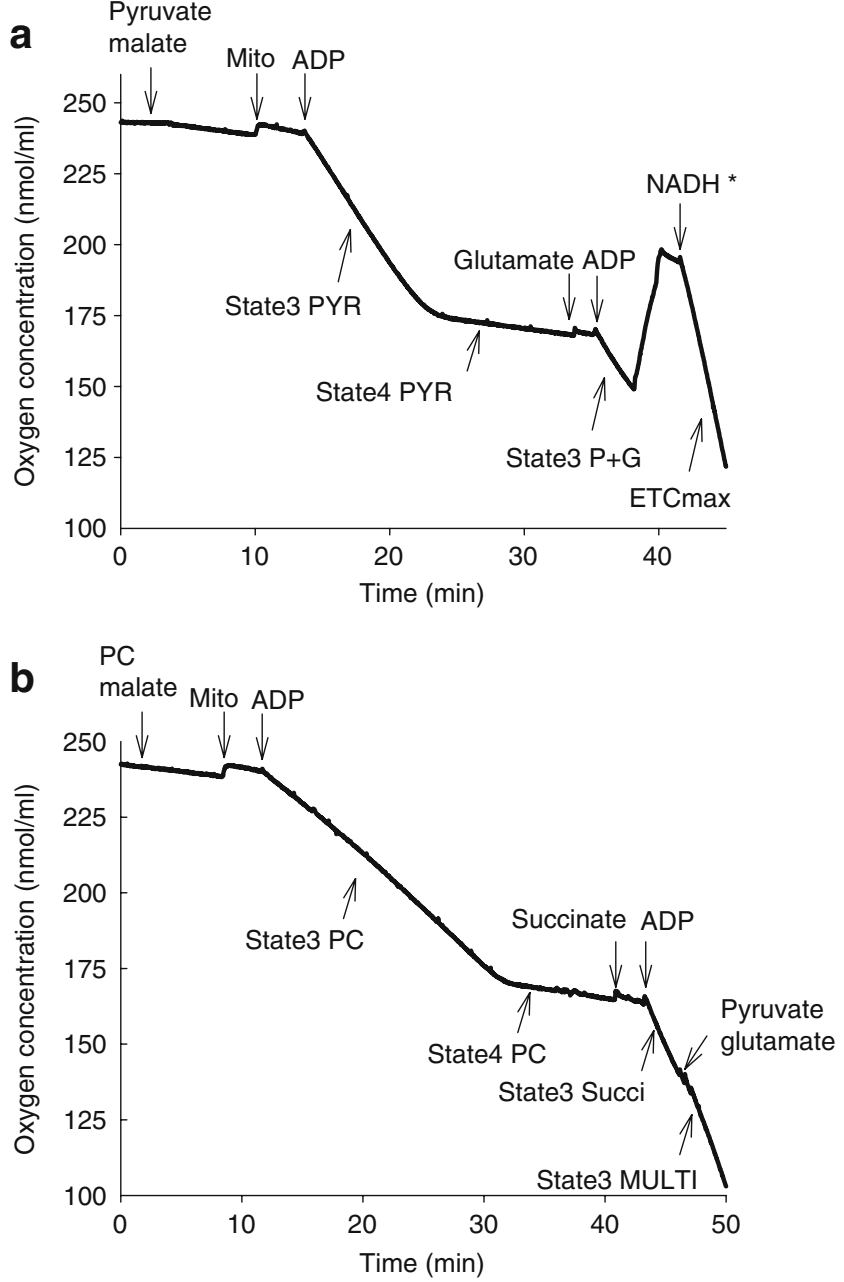

Fig. 1 Representative oxygraphic traces of mitochondrial oxygen consumption in isolated mitochondria for Protocol 1 (a) and Protocol 2 (b). Addition of substrates and isolated mitochondria (Mito) is indicated by arrows above the trace. Different parts of the trace are indicated by arrows below the trace. *Alamethicin and cytochrome $c$ added in addition to $\mathrm{NADH}$. The increased oxygen content prior to addition of NADH is due to aeration of the chamber in order to maintain adequate oxygen tension. PC, palmitoyl-L-carnitine; $\mathrm{P}+\mathrm{G}$, pyruvate + glutamate; Succi, succinate

(Molecular Probes, Carlsbad, CA, USA) as a trapper of $\mathrm{H}_{2} \mathrm{O}_{2}$, catalysed by horseradish peroxidase. Mitochondrial superoxide $\left(\mathrm{O}_{2}{ }^{-}\right)$is converted to $\mathrm{H}_{2} \mathrm{O}_{2}$ by addition of superoxide dismutase. $\mathrm{H}_{2} \mathrm{O}_{2}$ reacts with Amplex Red in a 1:1 stoichiometry yielding the fluorescent compound resorufin (excitation $560 \mathrm{~nm}$, emission $590 \mathrm{~nm}$ ), which is stable once formed. Fluorescence was measured continuously with a spectrofluorometer equipped with temperature control and magnetic stirring (Ratiomaster RCM; Photon Technology, Brunswick, NJ, USA). Isolated mitochondria were added to the measurement buffer containing (in $\mathrm{mmol} / \mathrm{l}$ unless otherwise stated): mannitol 225 ; sucrose 75 ; Tris 10 ; $\mathrm{KCl} 10 ; \mathrm{K}_{2} \mathrm{HPO}_{4} 10 ;$ EDTA $0.1 ; \mathrm{MgCl}_{2} \cdot\left(6 \mathrm{H}_{2} \mathrm{O}\right) 0.08$; Amplex Red 0.05; horseradish peroxidase $12 \mathrm{U} / \mathrm{ml}$; oligomycin $2 \mu \mathrm{g} / \mathrm{ml}$; and superoxide dismutase $90 \mathrm{U} / \mathrm{ml}$; $\mathrm{pH}$ 7.0.
Mitochondrial $\mathrm{H}_{2} \mathrm{O}_{2}$ release was determined after addition of succinate $(5.1 \mathrm{mmol} / \mathrm{l})$ plus rotenone $(2.1 \mu \mathrm{mol} / \mathrm{l})$ for measurement of mitochondrial ROS release in the presence of rotenone $\left(\mathrm{ROS}_{\mathrm{rot}}\right.$ ), which describes ROS production from complex III without blockage of forward electron flow. For measurement of mitochondrial ROS release in the presence of antimycin A ( ROS $\left._{\text {anti }}\right)$, antimycin A $(1.5 \mu \mathrm{g} / \mathrm{ml})$ was added, which blocked electron flow, causing maximal superoxide production from complex III. Four different concentrations of $\mathrm{H}_{2} \mathrm{O}_{2}$ were added to establish a standard curve.

Expression of genes encoding mitochondrial proteins and markers of mitochondrial biogenesis Total RNA was extracted from 1.0 to $2.5 \mathrm{mg}$ freeze-dried muscle with a reagent (PureZOL; Bio-Rad Laboratories, Sundbyberg, Sweden). RNA purity and concentration were determined with spectrometry. RNA $(1 \mu \mathrm{g})$ was used for transcription of $20 \mu \mathrm{l}$ cDNA with a kit (iScript cDNA Synthesis; BioRad). Real-time PCR amplification mixtures $(25 \mu \mathrm{l})$ contained $12.5 \mu 12 \times$ SYBR Green Supermix (Bio-Rad), $0.5 \mu \mathrm{l} 10 \mu \mathrm{mol} / \mathrm{l}$ forward and reverse primers respectively, and $11.5 \mu \mathrm{l}$ template cDNA and RNase-free water together. Control group and diabetes samples were run on the same plate to permit direct relative comparisons. Real-time PCR was performed with iCycler (Bio-Rad). A series of genes were determined, which included CPT1B, PDK4, COX1 (also known as $P T G S 1$ ), COX4 (also known as COX4I1), $P G C-1 \alpha$ (also known as PPARGC1A), PGC-1 $\beta$ (also known as PPARGC1B), PPARA, PPAR- $\beta$ (also known as PPARD), PPARG, PRC (also known as PPRCl) and $G A P D H$ was used as reference gene. mRNA relative levels were automatically analysed by the Normalised Expression Vandesompele $\left({ }^{\Delta \Delta} \mathrm{CT}\right.$ Advanced) tool in the software (iCycler; Bio-Rad).

Fibre-type distribution, UCP3 protein content and CS activity Fibre-type distribution was determined by electrophoresis as different isotypes of myosin heavy chain (MHC) I, MHC II and MHC IIx as previously described [32]. UCP3 was determined in fourteen participants (type 2 diabetes $n=6$, control $n=8$ ). Determination of UCP3 protein content was analysed in duplicate as described in detail previously [33]. The U CS activity in the mitochondrial rich solutions $\left(\mathrm{CS}_{\text {mito }}\right)$ and of $\mathrm{CS}$ per $\mathrm{g}$ muscle wet weight $\left(\mathrm{CS}_{\text {muscle }}\right)$ was determined spectrophotometrically at $25^{\circ} \mathrm{C}$ as described by Alp et al. [34]. Intrinsic mitochondrial respiration and $\mathrm{H}_{2} \mathrm{O}_{2}$ production in isolated mitochondria were determined by relating measures to $\mathrm{CS}_{\text {mito }}$. Respiration per kg wet weight was calculated by multiplying intrinsic respiration by $\mathrm{CS}_{\text {muscle }}$ activity.

Statistics Data are presented as means \pm SEM. A software package (Systat software, Richmond, CA, USA) was used 
Table 1 Anthropometric and physical fitness characteristics

\begin{tabular}{|c|c|c|c|c|}
\hline \multirow[t]{2}{*}{ Characteristics } & \multicolumn{2}{|l|}{ Pre-training } & \multicolumn{2}{|l|}{ Post-training } \\
\hline & Type 2 diabetes & Control & Type 2 diabetes & Control \\
\hline$n$ & 13 & 14 & 12 & 12 \\
\hline Age (years) & $52.3 \pm 1.3$ & $52.6 \pm 1.6$ & $52.5 \pm 1.4$ & $52.8 \pm 1.7$ \\
\hline Weight (kg) & $108 \pm 2$ & $109 \pm 3$ & $108 \pm 2$ & $108 \pm 4^{\mathrm{a}}$ \\
\hline BMI $\left(\mathrm{kg} / \mathrm{m}^{2}\right)$ & $33.5 \pm 1.0$ & $32.9 \pm 0.7$ & $33.4 \pm 1.0$ & $32.6 \pm 0.8^{\mathrm{a}}$ \\
\hline FFM (kg) & $72.7 \pm 1.9$ & $74.1 \pm 2.0$ & $74.3 \pm 1.7$ & $75.5 \pm 2.2$ \\
\hline $\begin{array}{l}\dot{V} \mathrm{O}_{2 \text { peak }} \\
\quad\left(\mathrm{ml} \mathrm{O}_{2} / \mathrm{min}\right)\end{array}$ & $2,865 \pm 108$ & $3,042 \pm 164$ & $3,203 \pm 113^{\mathrm{a}}$ & $3,688 \pm 193^{\mathrm{a}}$ \\
\hline $\begin{array}{l}\dot{V} \mathrm{O}_{2 \text { peak }} \\
\left(\mathrm{ml} \mathrm{O}_{2} \min ^{-1} \mathrm{FFM}^{-1}\right) \\
\text { Fibre type composition }\end{array}$ & $39.5 \pm 1.4$ & $41.8 \pm 2.3$ & $43.2 \pm 1.5^{\mathrm{a}}$ & $48.1 \pm 2.7^{\mathrm{a}}$ \\
\hline MHC I & $36.4 \pm 2.4$ & $34.7 \pm 3.4$ & $36.0 \pm 3.1$ & $35.6 \pm 2.4$ \\
\hline MHC II & $56.8 \pm 2.8$ & $59.2 \pm 3.4$ & $57.0 \pm 2.7$ & $61.4 \pm 2.6$ \\
\hline MHC IIx & $6.7 \pm 1.6$ & $6.1 \pm 1.1$ & $7.1 \pm 1.4$ & $2.9 \pm 0.8$ \\
\hline
\end{tabular}

for all statistical calculations. Differences between groups pre- and post-training and the effect of training were evaluated by two-way ANOVA with repeated measures. Significant main effects or interactions were further analysed by Bonferroni post hoc tests. Correlations were examined by calculation of Pearson's correlation coefficients. Significance was accepted at $p \leq 0.05$.

\section{Results}

Participant characteristics Patients with type 2 diabetes and control participants were matched with respect to age, BMI and $\dot{V} \mathrm{O}_{2 \text { peak }}$ (Table 1). Training caused a significant increase in $\dot{V} \mathrm{O}_{2 \text { peak }}$ in both groups, with no difference in increase between groups (type 2 diabetes $12 \pm 4 \%$ vs control $16 \pm 2 \%, p=0.4$ ) (Table 1). Training also resulted in a significant decrease in weight and BMI, but only in control participants (Table 1).

Insulin sensitivity and blood profile $R_{\mathrm{d}}$ was significantly lower in type 2 diabetes patients both before and after training (Table 2). Training caused a significant increase in $R_{\mathrm{d}}$ in type 2 diabetes $(13 \%, p=0.001)$ and control participants $(22 \%, p<0.001)$. Before and after training, type 2 diabetes patients had significantly higher $\mathrm{HbA}_{1 \mathrm{c}}$ and plasma glucose levels. Neither plasma glucose levels nor $\mathrm{HbA}_{1 \mathrm{c}}$ were significantly altered in response to training (Table 2). $R_{\mathrm{d}}$ did not correlate with intrinsic mitochondrial respiratory function or mitochondrial ROS release.

Respiration in isolated mitochondria When expressed per $\mathrm{kg}$ muscle mass, training caused a significant $\sim 63 \%$ increase in respiration with complex I-supported substrates (pyruvate and glutamate) in both groups. Training also increased $\mathrm{ETC}_{\max }$ and mitochondrial lipid oxidation (palmitoyl-Lcarnitine + malate) in type 2 diabetes patients (Fig. 2a, b). Similar changes were observed with other substrates, but the changes were not statistically significant (Fig. 2b). There was no difference between groups in the respiratory measures (Fig. 2).

When expressed per mitochondrial density (i.e. CS activity), training increased intrinsic State 3 PYRUVATE $(p=$ $0.02)$ and State $3_{\text {Pyruvate+Glutamate }}(p=0.03)$, and decreased State4 $4_{\text {PALMITOYL-L-CARNITINE }}(p=0.005)$ and State $4_{\text {PYRUVATE }}(p=0.01)$ in the whole groups of participants (Table 3). When divided into groups, training tended to increase State $3_{\text {PYRUVATE }}(p=0.07)$ and State $3_{\text {PYRUVATE }}$ GLUTAMATE $(p=0.06)$ in control participants, but there were otherwise no significant changes in intrinsic respiratory
Table 2 Clinical data and blood profile

Values are mean $\pm \mathrm{SE}$

a $p<0.05$ vs type 2 diabetes;

${ }^{\mathrm{b}} p<0.05$ vs pre-training

FFM, fat-free-mass

\begin{tabular}{|c|c|c|c|c|}
\hline \multirow[t]{2}{*}{ Variable } & \multicolumn{2}{|l|}{ Pre-training } & \multicolumn{2}{|l|}{ Post-training } \\
\hline & Type 2 diabetes & Control & Type 2 diabetes & Control \\
\hline$n$ & 13 & 14 & 12 & 12 \\
\hline Diabetes duration (years) & $3.7 \pm 0.8$ & - & - & - \\
\hline$R_{\mathrm{d}}\left(\mathrm{mg} \min ^{-1}[\mathrm{~kg} \mathrm{FFM}]^{-1}\right)$ & $6.5 \pm 0.8$ & $10.3 \pm 0.7^{\mathrm{a}}$ & $7.3 \pm 0.9^{\mathrm{b}}$ & $12.5 \pm 1.2^{\mathrm{a}, \mathrm{b}}$ \\
\hline Fasting plasma glucose $(\mathrm{mmol} / \mathrm{l})$ & $9.4 \pm 0.5$ & $5.7 \pm 0.1^{\mathrm{a}}$ & $9.2 \pm 0.5$ & $5.5 \pm 0.1^{\mathrm{a}}$ \\
\hline $\mathrm{HbA}_{1 \mathrm{c}}(\%)$ & $7.2 \pm 0.3$ & $5.2 \pm 0.1^{\mathrm{a}}$ & $7.0 \pm 0.3$ & $5.3 \pm 0.1^{\mathrm{a}}$ \\
\hline
\end{tabular}



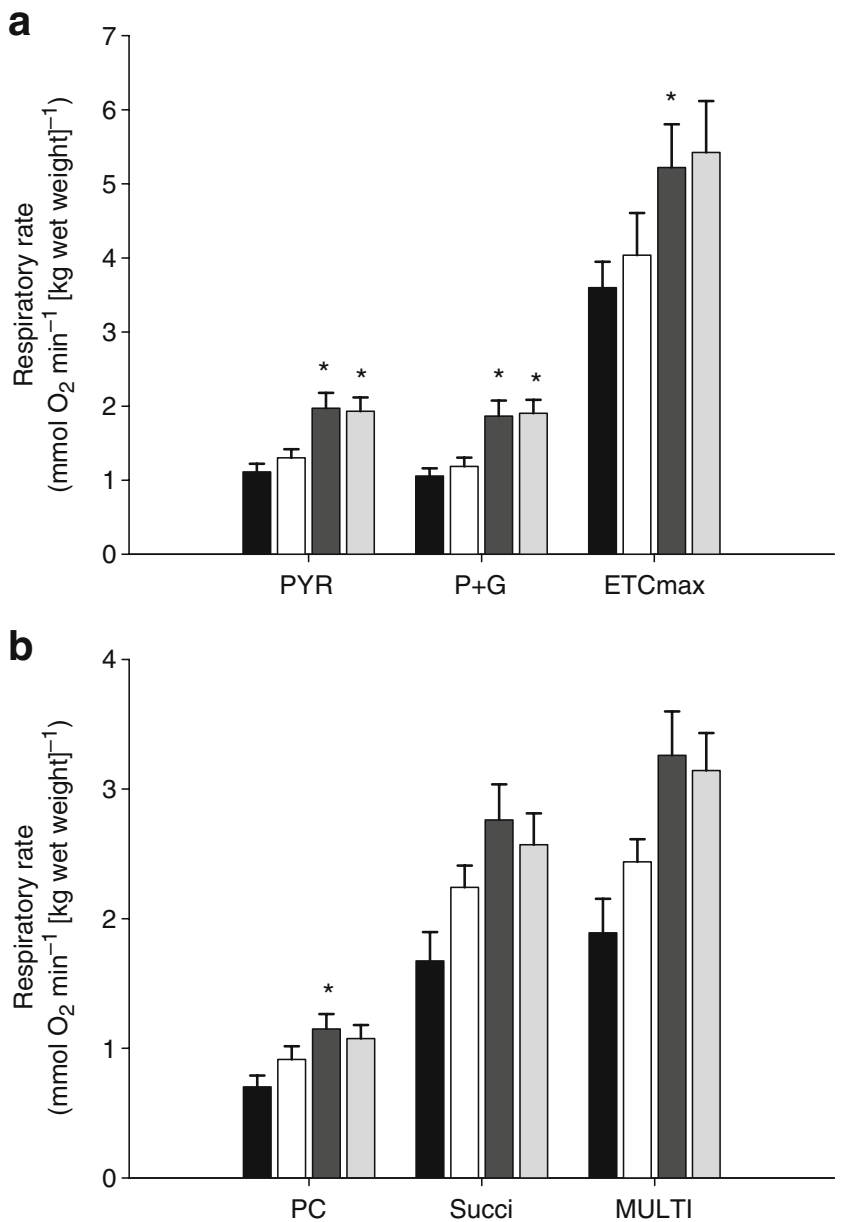

Fig. 2 Respiration in isolated mitochondria. State 3 respiration in isolated mitochondria was calculated per $\mathrm{kg}$ wet weight of muscle for a pyruvate + malate $(\mathrm{PYR})$, pyruvate + malate + glutamate $(\mathrm{P}+\mathrm{G})$ and cytochrome $c+$ alamethicin + NADH (ETCmax), and b palmitoyl-L-carnitine+malate (PC), palmitoyl-L-carnitine + malate+succinate (Succi) and palmitoyl-Lcarnitine + malate + succinate + pyruvate + glutamate (MULTI). Black bars, type 2 diabetes participants pre-training; white bars, control participants pre-training; dark grey bars, type 2 diabetes participants post-training; light grey bars, control participants post-training. Data are mean \pm SEM. $* p<0.05$ vs pre-training within group

measures within groups (Table 3). Training caused a significant increase in RCI pyruvate and RCI palmitoyl-Lcarnitine in the whole group of participants. Within groups, type 2 diabetes patients also had a significant increase in RCI palmitoyl-L-carnitine (Table 3).

Mitochondrial ROS release Pre-training type 2 diabetes patients tended to have a higher succinate-supported mitochondrial $\operatorname{ROS}_{\mathrm{rot}}(p=0.10)$, but there was no difference after addition of antimycin $\mathrm{A}\left(\mathrm{ROS}_{\mathrm{anti}}\right)$ (Fig. 3). Training tended to decrease $\mathrm{ROS}_{\text {rot }}$ in the whole group of participants $(p=0.10)$, but had no significant effect on $\operatorname{ROS}_{\text {anti }}$.

CS activity, UCP3 protein content and fibre-type composition Training caused a significant increase in muscle CS activity in both groups (type 2 diabetes pre $60.8 \pm 5.6$, post $98.0 \pm 9.6$; control pre $78.5 \pm 4.3$, post $102.4 \pm 9.1 \mathrm{U} / \mathrm{mg}$ dry weight; $p<$ 0.01 ), but there was no difference between groups either before or after training. Due to limited material, UCP3 protein content was only determined in 14 participants $(n=6$ type 2 diabetes, $n=8$ control). In the whole group of participants, UCP3 protein content increased $\sim 69 \%$ in response to training. When divided into groups, only control participants showed a significant increase in UCP3 protein content (control $71 \pm$ $26 \%, p<0.001$; type 2 diabetes $67 \pm 65 \%, p=0.4$ ). There was no significant difference between groups in fibre-type composition. Training caused a $60 \%$ decrease in MHCIIx in control participants. However, this decrease was not statistically significant (Table 1, Fig. 4).

Gene expression Pre-training type 2 diabetes patients had significantly higher expression of PDK4 than control participants, but there were no other differences in gene expression between groups. Training increased mRNA of PPARG significantly in type 2 diabetes patients $(p=0.02)$ and in control participants $(p=0.001)$ (Fig. 5b). Interestingly, mRNA of PDK4 ( $p<0.001), C O X 1 \quad(p=0.008)$ and COX4 $(p=0.02)$ increased significantly in control participants after training (Fig. 5a), but not in type 2 diabetes patients.

\section{Discussion}

The main result of the present study is that 10 weeks of aerobic training resulted in a significant increase in mitochondrial respiratory capacity when determined per muscle weight. When respiration was related to a marker of mitochondrial content (intrinsic), training resulted in increased complex I-supported respiration and a decrease in State4 respiration. Training also increased expression of genes encoding mitochondrial ETC proteins and of PPARG, suggesting increased mitochondrial biogenesis. There was no difference in training adaptability between type 2 diabetes and control participants. This demonstrates that aerobic training improves muscle oxidative function regardless of the degree of insulin resistance. Another interesting finding was that mitochondrial ROS release tended to be higher in type 2 diabetes patients than in control participants and tended to decrease in response to training.

Effect of training on mitochondrial respiration and mitochondrial biogenesis The main purpose of the present study was to investigate the effect of physical training on several mitochondrial variables in obese participants with and without type 2 diabetes, and matched for physical fitness, obesity and age. Training resulted in a large (about 
Table 3 Mitochondrial respiratory function

Values are mean $\pm \mathrm{SE}$

${ }^{\mathrm{a}} p<0.05$ vs pre-training; ${ }^{\mathrm{b}} p<0.05$

pre- vs post-training in the whole group of participants

${ }^{\mathrm{c}} n=11,{ }^{\mathrm{d}} n=8,{ }^{\mathrm{e}} n=12$ and ${ }^{\mathrm{f}} n=10$

type 2 diabetes in the pre-training

state; ${ }^{\mathrm{g}} n=9$ controls in

pre-training state

ETCmax, alamethicin+

cytochrome $c+\mathrm{NADH}$; P:O

ratio, ratio of $\mathrm{ADP}$ consumed:

oxygen utilised; State $3_{\mathrm{G}+\mathrm{P}}$,

State 3 glutamate + pyruvate +

malate; State $3_{\mathrm{PC}+\mathrm{S}}$, State3

palmitoyl-L-carnitine + succinate;

State $3_{\text {MULTI }}$, State 3 pyruvate+

malate+ glutamate + palmitoyl-

L-carnitine + succinate

\begin{tabular}{|c|c|c|c|c|}
\hline \multirow[t]{2}{*}{ Variable } & \multicolumn{2}{|l|}{ Pre-training } & \multicolumn{2}{|l|}{ Post-training } \\
\hline & Type 2 diabetes & Control & Type 2 diabetes & Control \\
\hline$n$ & 13 & 12 & 12 & 12 \\
\hline \multicolumn{5}{|c|}{ Pyruvate + malate } \\
\hline State $3^{\mathrm{b}}$ & $79.7 \pm 3.8$ & $73.2 \pm 4.7$ & $87.1 \pm 3.7$ & $82.4 \pm 3.1$ \\
\hline State $4^{\mathrm{b}, \mathrm{c}, \mathrm{g}}$ & $7.5 \pm 0.8$ & $6.7 \pm 1.2$ & $5.5 \pm 0.8$ & $4.9 \pm 0.4$ \\
\hline $\mathrm{RCI}^{\mathrm{b}, \mathrm{c}, \mathrm{g}}$ & $11.8 \pm 1.4$ & $9.9 \pm 0.9$ & $14.3 \pm 1.4$ & $13.9 \pm 0.6$ \\
\hline $\mathrm{P}: \mathrm{O}$ ratio $^{\mathrm{e}, \mathrm{g}}$ & $2.75 \pm 0.04$ & $2.61 \pm 0.08$ & $2.58 \pm 0.03$ & $2.62 \pm 0.02$ \\
\hline \multicolumn{5}{|c|}{ Palmitoyl-L-carnitine + malate } \\
\hline State $3^{\mathrm{f}, \mathrm{g}}$ & $47.8 \pm 2.1$ & $51.0 \pm 1.7$ & $51.6 \pm 3.0$ & $46.3 \pm 2.5$ \\
\hline State $4^{\mathrm{b}, \mathrm{f}, \mathrm{g}}$ & $7.4 \pm 0.8$ & $6.6 \pm 0.40$ & $5.3 \pm 0.3$ & $5.1 \pm 0.3$ \\
\hline $\mathrm{RCI}^{\mathrm{b}, \mathrm{d}, \mathrm{g}}$ & $5.9 \pm 0.4$ & $6.3 \pm 0.3$ & $8.7 \pm 0.6^{\mathrm{b}}$ & $7.7 \pm 0.3$ \\
\hline $\mathrm{P}: \mathrm{O}$ ratio $^{\mathrm{d}, \mathrm{g}}$ & $2.47 \pm 0.04$ & $2.49 \pm 0.03$ & $2.42 \pm 0.03$ & $2.49 \pm 0.04$ \\
\hline State $3_{\mathrm{G}+\mathrm{P}}^{\mathrm{b}}$ & $76.4 \pm 4.5$ & $66.7 \pm 6.0$ & $84.9 \pm 4.4$ & $81.2 \pm 2.9$ \\
\hline State $3_{\mathrm{PC}+\mathrm{S}}^{\mathrm{f}, \mathrm{g}}$ & $116 \pm 9$ & $128 \pm 4$ & $123 \pm 5$ & $111 \pm 6$ \\
\hline State $3_{\text {MULTI }}{ }^{\mathrm{f}, \mathrm{g}}$ & $129 \pm 12$ & $140 \pm 7$ & $145 \pm 6$ & $136 \pm 7$ \\
\hline ETCmax & $260 \pm 16$ & $243 \pm 26$ & $245 \pm 28$ & $224 \pm 14$ \\
\hline
\end{tabular}

$63 \%$ ) increase in muscle oxidative capacity shown by increased State 3 respiration per $\mathrm{kg}$ muscle weight. These results are in accordance with a recent study showing that in vivo mitochondrial function increased in type 2 diabetic and control participants [26]. The present study expands on this finding by showing that the adaptation seems to be most pronounced in the mitochondrial ability to use complex I-supporting substrates, since the most significant adaptation was when pyruvate and glutamate were used as

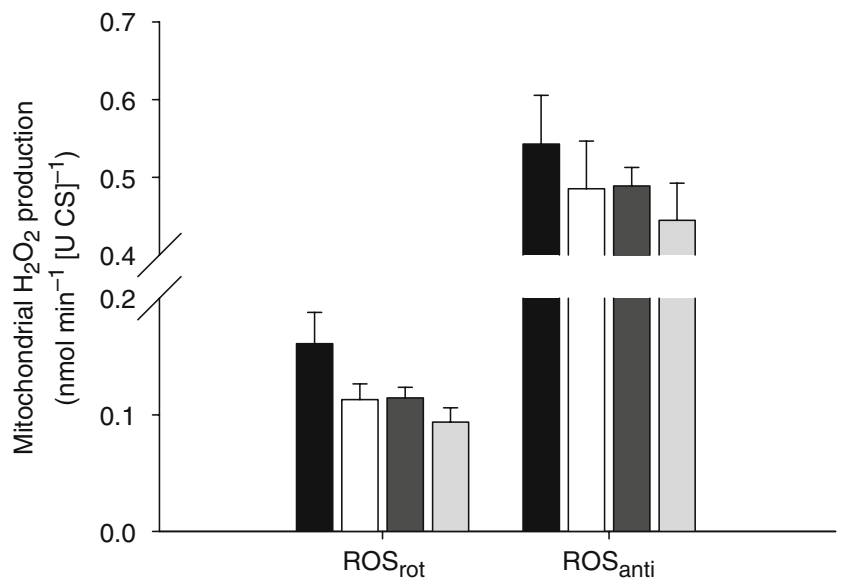

Fig. 3 Mitochondrial $\mathrm{H}_{2} \mathrm{O}_{2}$ release. Hydrogen peroxide $\left(\mathrm{H}_{2} \mathrm{O}_{2}\right)$ release in isolated mitochondria with succinate+rotenone+oligomycin $\left(\mathrm{ROS}_{\text {rot }}\right)$ and succinate + rotenone + oligomycin + antimycin A $\left(\mathrm{ROS}_{\text {anti }}\right)$ from skeletal muscle of obese control participants and obese patients with type 2 diabetes. Black bars, type 2 diabetes participants pretraining; white bars, control participants pre-training; dark grey bars, type 2 diabetes participants post-training; light grey bars, control participants post-training. Values were from 12 type 2 diabetes patients and 12 (pre-training) and nine (post-training) control participants. Data are mean \pm SEM substrates (Fig. 2a). As suggested by Meex et al. [26], an increase in muscle oxidative capacity is most likely to be caused by increased mitochondrial content and/or increased intrinsic mitochondrial activity. In the present study, CS activity, a marker of mitochondrial content, increased by $\sim 50 \%$ in the whole group. Furthermore, even though biopsies were taken $48 \mathrm{~h}$ after the last training session, expression of genes encoding several mitochondrial proteins was significantly increased, supporting a sustained increase in mitochondrial biogenesis (Fig. 5a, b). Even though the response to training in some cases seemed to be more pronounced in control participants (expression of PDK4, COX1 and COX4; MHCIIx protein levels), collectively the results suggest that the higher degree of insulin resistance in type 2 diabetes patients does not alter the adaptation of muscle oxidative capacity and mitochondrial functionality.

An interesting finding of this study was that intrinsic mitochondrial respiration with NADH-generating substrates (pyruvate and glutamate) increased about 11\% in response to training, whereas that with other substrate combinations $\left(\mathrm{ETC}_{\max }\right.$, State $3_{\text {PALMITOYL-L-CARNITINE, }}$ State 3 PAlmitoyl-L-CARnitine+Succinate, State $3_{\text {MUlti }}$ ) was unchanged. The increased State $3_{\text {Pyruvate }}$ and

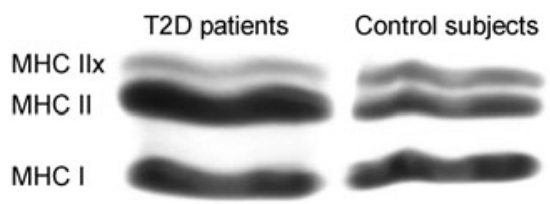

Fig. 4 Representative blot showing MHC from one participant per group 

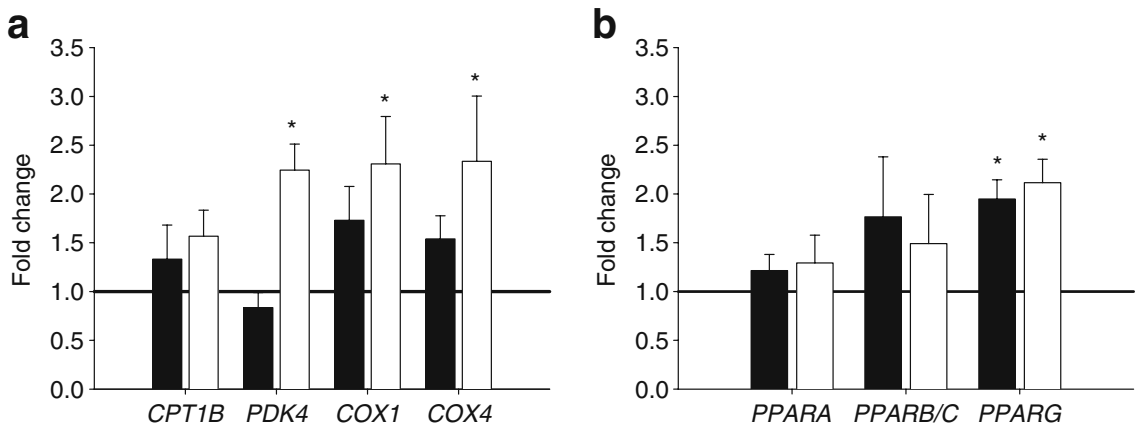

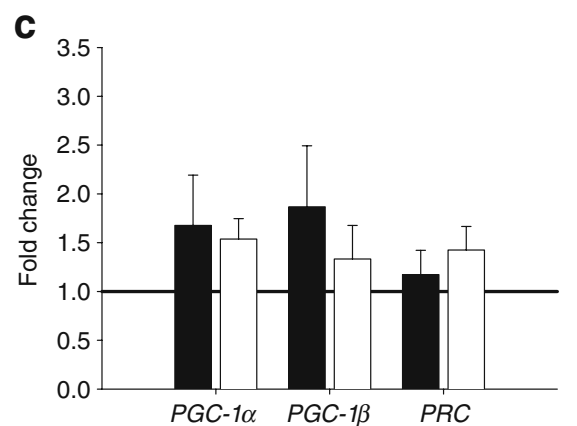

Fig. 5 Training-induced changes in expression of genes encoding markers of mitochondrial biogenesis and mitochondrial proteins, shown as changes in mRNA of mitochondrial proteins (a) and of proteins inducing mitochondrial biogenesis $(\mathbf{b}, \mathbf{c})$. Black bars, type 2

State $3_{\text {PYRUVATE+GLUTAMATE }}$ without any change in $\mathrm{ETC}_{\max }$ may be related to improved provision of NADH to ETC from the tricarboxylic acid cycle or from the reactions mediated by pyruvate dehydrogenase and glutamate dehydrogenase. An increase in State3 respiration in response to increased training status is in contrast to what we [35] and others [33] have reported previously in young, trained and untrained participants. Values of State $3_{\text {PYRUVATE }}$ obtained prior to training were about $15 \%$ lower in this study than previously observed in young non-obese participants [35]. However, after training State $3_{\text {PYRUVATE }}$ increased to the same level as in young participants. This difference between young lean and middle-aged obese participants in intrinsic mitochondrial respiration is in accordance with previous reports [33] and is most likely to be due to differences in physical fitness and/or age.

Mitochondrial ROS release and the effect of training Previous studies have suggested that increased cytosolic concentration of free radicals have an inhibitory effect on the insulin signalling cascade [16]. A main purpose of the present study was to investigate the hypothesis that insulin resistance is associated with increased mitochondrial ROS release. We showed that type 2 diabetes patients tended to have higher $\mathrm{ROS}_{\text {rot }}$, which training tended to decrease. A recent study showed that type 2 diabetes patients had higher mitochondrial ROS release than matched obese control participants [19]. It has also been shown that type 2 diabetes patients had significantly higher ROS release than lean participants when related to ATP production, but similar ROS release when related to mitochondrial protein [19]. Similarly to the protocol used by Abdul-Ghani et al.

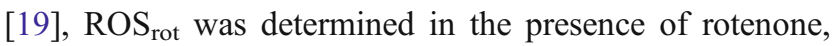
which inhibits the unphysiological backflow of electrons from complex II to complex I. The fate of the electrons is therefore: (1) to be donated to oxygen, forming water in complex IV; or (2) to be donated to oxygen, forming superoxide in complex III. Although this in vitro model diabetes participants; white bars, control participants; horizontal lines, pre-training values. Values are from eight type 2 diabetes patients and eight control participants. Data are mean \pm SEM. ${ }^{*} p<0.05$ vs pretraining within group

may not correspond to conditions in vivo, the results demonstrate an increased potential for ROS release in ETC, which will probably have an impact on ROS production in vivo. The excessive supply of substrate in type 2 diabetes (hyperglycaemia and hyperlipidaemia) may be an additional factor that increases ROS production in vivo.

One mitochondrial protein related to mitochondrial ROS production is UCP3. Previous studies have suggested that UCP3 acts as a protective mechanism against mitochondrial ROS production [36]. Indeed, training did cause a significant $72 \%$ increase in UCP3 protein in the whole group of participants. Moreover, as described above, it also caused a $\sim 50 \%$ increase in mitochondrial content (CS activity). Thus, UCP3 protein levels per mitochondrion would also have been increased and may in part explain the tendency towards decreased intrinsic mitochondrial ROS release. However, further research is needed to verify that UCP3 contributes to uncoupling, thereby leading to lower ROS production in humans.

The potential link between insulin resistance and mitochondrial function During the last decade there has been an increased interest in the potential link between mitochondrial function in skeletal muscle and insulin sensitivity in obesity and type 2 diabetes. However, only few studies have assessed intrinsic mitochondrial function. Of these, some have shown lower intrinsic mitochondrial function $[6,12,13]$, whereas others have been unable to detect a difference between type 2 diabetes patients and control participants [9-11, 14, 15, 37]. One study, moreover, showed higher lipid oxidation in isolated mitochondria in patients with type 2 diabetes [38]. Recently, we reported impaired mitochondrial respiration in skeletal muscle of patients with type 2 diabetes when measured as $\mathrm{ETC}_{\max }$ and State $3_{\text {PYRUVATE }}$ respiration [13]. However, in that study, the level of physical activity and aerobic capacity was not determined. In the present study, groups had similar aerobic capacities and there was no difference in 
any intrinsic respiratory measures. We have also demonstrated that physical training increases intrinsic mitochondrial respiration. We therefore suspect that the difference in intrinsic mitochondrial respiration reported in our previous study was due to lower physical fitness in type 2 diabetes patients than in the control participants.

In summary, the present study shows that aerobic training increases muscle oxidative capacity and intrinsic mitochondrial State3 respiration, decreases State4 respiration and increases mitochondrial biogenesis in middle-aged obese men with and without type 2 diabetes. Increased State 3 and decreased State4 respiration represent an increase in mitochondrial functionality that is most likely to be related to an improvement in mitochondrial substrate handling. Type 2 diabetes patients were significantly more insulin-resistant than control participants matched for age, obesity and physical activity, but there were no differences between groups in mitochondrial respiration. These findings refute the hypothesis that the additional insulin resistance seen in obese type 2 diabetic patients vs those with obesity alone is caused by low intrinsic mitochondrial activity or low oxidative capacity. However, the possibility that a combination of low muscle oxidative capacity and substrate overload (hyperglycaemia, hyperlipidaemia) creates a condition of increased ROS production and insulin resistance remains to be investigated.

Acknowledgements The authors wish to express their gratitude to L. Hansen, C. B. Olsen, C. Christensen, R. Kraunsøe and B. Jørgensen for excellent technical assistance and to J. Nielsen and M. Antvorskov for supervising the lab training. The work was supported by grants from the Danish Medical Research Council, the Novo Nordisk Foundation, the Danish Diabetes Association, Nordea Foundation, AP Møller Foundation (Fonden til Lægevidenskabens Fremme) and the Swedish Research Council.

Duality of interest The authors declare that there is no duality of interest associated with this manuscript.

\section{References}

1. Eriksson J, Franssilakallunki A, Ekstrand A et al (1989) Early metabolic defects in persons at increased risk for non-insulindependent diabetes-mellitus. N Engl J Med 321:337-343

2. Kelley DE, Mandarino LJ (2000) Fuel selection in human skeletal muscle in insulin resistance: a reexamination. Diabetes 49: 677-683

3. Szendroedi J, Schmid AI, Chmelik M et al (2007) Muscle mitochondrial ATP synthesis and glucose transport/phosphorylation in type 2 diabetes. PLoS Med 4:858-867

4. De Feyter HM, van den Broek NM, Praet SF, Nicolay K, van Loon LJ, Prompers JJ (2008) Early or advanced stage type 2 diabetes is not accompanied by in vivo skeletal muscle mitochondrial dysfunction. Eur J Endocrinol 158:643-653

5. Brons C, Jensen CB, Storgaard H et al (2008) Mitochondrial function in skeletal muscle is normal and unrelated to insulin action in young men born with low birth weight. J Clin Endocrinol Metab 93:3885-3892

6. Phielix E, Schrauwen-Hinderling VB, Mensink M et al (2008) Lower intrinsic ADP-stimulated mitochondrial respiration underlies in vivo mitochondrial dysfunction in muscle of male type 2 diabetic patients. Diabetes 57:2943-2949

7. Schrauwen-Hinderling VB, Kooi ME, Hesselink MKC et al (2007) Impaired in vivo mitochondrial function but similar intramyocellular lipid content in patients with type 2 diabetes mellitus and BMI-matched control subjects. Diabetologia 50: $113-120$

8. Petersen KF, Dufour S, Befroy D, Garcia R, Shulman GI (2004) Impaired mitochondrial activity in the insulin-resistant offspring of patients with type 2 diabetes. N Engl J Med 350:664-671

9. Boushel R, Gnaiger E, Schjerling P, Skovbro M, Kraunsoe R, Dela F (2007) Patients with type 2 diabetes have normal mitochondrial function in skeletal muscle. Diabetologia 50: 790-796

10. Rabol R, Hojberg PMV, Almdal T et al (2009) Effect of hyperglycemia on mitochondrial respiration in type 2 diabetes. $\mathrm{J}$ Clin Endocrinol Metab 94:1372-1378

11. Kelley DE, He J, Menshikova EV, Ritov VB (2002) Dysfunction of mitochondria in human skeletal muscle in type 2 diabetes. Diabetes 51:2944-2950

12. Ritov VB, Menshikova EV, He J, Ferrell RE, Goodpaster BH, Kelley DE (2005) Deficiency of subsarcolemmal mitochondria in obesity and type 2 diabetes. Diabetes 54:8-14

13. Mogensen M, Sahlin K, Fernstrom M et al (2007) Mitochondrial respiration is decreased in skeletal muscle of patients with type 2 diabetes. Diabetes 56:1592-1599

14. Asmann YW, Stump CS, Short KR et al (2006) Skeletal muscle mitochondrial functions, mitochondrial DNA copy numbers, and gene transcript profiles in type 2 diabetic and nondiabetic subjects at equal levels of low or high insulin and euglycemia. Diabetes 55:3309-3319

15. Nair KS, Bigelow ML, Asmann YW et al (2008) Asian Indians have enhanced skeletal muscle mitochondrial capacity to produce ATP in association with severe insulin resistance. Diabetes 57:1166-1175

16. Maddux BA, See W, Lawrence JC, Goldfine AL, Goldfine ID, Evans JL (2001) Protection against oxidative stress-induced insulin resistance in rat L6 muscle cells by micromolar concentrations of alpha-lipoic acid. Diabetes 50:404-410

17. Evans JL, Goldfine ID, Maddux BA, Grodsky GM (2002) Oxidative stress and stress-activated signaling pathways: a unifying hypothesis of type 2 diabetes. Endocr Rev 23:599-622

18. Anderson EJ, Lustig ME, Boyle KE et al (2009) Mitochondrial $\mathrm{H}_{2} \mathrm{O}_{2}$ emission and cellular redox state link excess fat intake to insulin resistance in both rodents and humans. J Clin Investig 119:573-581

19. Abdul-Ghani MA, Jani R, Chavez A, Molina-Carrion M, Tripathy D, DeFronzo RA (2009) Mitochondrial reactive oxygen species generation in obese non-diabetic and type 2 diabetic participants. Diabetologia 52:574-582

20. Ristow M, Zarse K, Oberbach A et al (2009) Antioxidants prevent health-promoting effects of physical exercise in humans. Proc Natl Acad Sci U S A 106:8665-8670

21. Loh K, Deng HY, Fukushima A et al (2009) Reactive oxygen species enhance insulin sensitivity. Cell Metab 10:260-272

22. Turrens JF (2003) Mitochondrial formation of reactive oxygen species. J Physiol-London 552:335-344

23. Venditti P, Masullo P, Di Meo S (1999) Effect of training on $\mathrm{H}_{2} \mathrm{O}_{2}$ release by mitochondria from rat skeletal muscle. Arch Biochem Biophys 372:315-320

24. Dela F, Larsen JJ, Mikines KJ, Ploug T, Petersen LN, Galbo H (1995) Insulin-stimulated muscle glucose clearance in patients 
with niddm - effects of one-legged physical-training. Diabetes 44:1010-1020

25. Bruce CR, Hawley JA (2004) Improvements in insulin resistance with aerobic exercise training: a lipocentric approach. Med Sci Sports Exerc 36:1196-1201

26. Meex RC, Schrauwen-Hinderling VB, Moonen-Kornips E et al (2010) Restoration of muscle mitochondrial function and metabolic flexibility in type 2 diabetes by exercise training is paralleled by increased myocellular fat storage and improved insulin sensitivity. Diabetes 59:572-579

27. Toledo FGS, Menshikova EV, Ritov VB et al (2007) Effects of physical activity and weight loss on skeletal muscle mitochondria and relationship with glucose control in type 2 diabetes. Diabetes $56: 2142-2147$

28. Fritz T, Kramer DK, Karlsson HKR et al (2006) Low-intensity exercise increases sketelal muscle protein expression of PPAR delta and UCP3 in type 2 diabetic patients. Diab/Metab Res Rev 22:492-498

29. De Filippis E, Alvarez G, Berria R et al (2008) Insulin-resistant muscle is exercise resistant: evidence for reduced response of nuclear-encoded mitochondrial genes to exercise. Am J Physiol Endocrinol Metab 294:E607-E614

30. Mogensen M, Vind BF, Hojlund K, Beck-Nielsen H, Sahlin K (2009) Maximal lipid oxidation in patients with type 2 diabetes is normal and shows an adequate increase in response to aerobic training. Diabetes Obes Metab 11:874-883

31. Tonkonogi M, Sahlin K (1997) Rate of oxidative phosphorylation in isolated mitochondria from human skeletal muscle: effect of training status. Acta Physiol Scand 161:345-353
32. Hansen EA, Andersen JL, Nielsen JS, Sjogaard G (2002) Muscle fibre type, efficiency, and mechanical optima affect freely chosen pedal rate during cycling. Acta Physiol Scand 176:185194

33. Tonkonogi M, Fernstrom M, Walsh B et al (2003) Reduced oxidative power but unchanged antioxidative capacity in skeletal muscle from aged humans. Pflugers Arch 446:261269

34. Alp PR, Newsholme EA, Zammit VA (1976) Activities of citrate synthase and NAD+-linked and NADP+-linked isocitrate dehydrogenase in muscle from vertebrates and invertebrates. Biochem J 154:689-700

35. Mogensen M, Bagger M, Pedersen PK, Fernstrom M, Sahlin K (2006) Cycling efficiency in humans is related to low UCP3 content and to type I fibres but not to mitochondrial efficiency. J Physiol-London 571:669-681

36. Nabben M, Hoeks J, Glatz JFC, Briede JJ, Hesselink MKC, Schrauwen P (2008) UCP3 and mitochondrial ROS production in cardiac and skeletal muscle. Cardiovasc Drugs Ther 22:151

37. Larsen S, Ara I, Rabol R, et al (2009) Are substrate use during exercise and mitochondrial respiratory capacity decreased in arm and leg muscle in type 2 diabetes? Diabetologia 52:1400-1408

38. Bandyopadhyay GK, Yu JG, Ofrecio J, Olefsky JM (2006) Increased malonyl-CoA levels in muscle from obese and type 2 diabetic subjects lead to decreased fatty acid oxidation and increased lipogenesis; thiazolidinedione treatment reverses these defects. Diabetes 55:2277-2285 